\title{
Expression of RUNX3 gene in pancreatic adenocarcinoma and its clinical significance
}

\author{
L.-N. Xue ${ }^{1 *}$, F.H. Bai ${ }^{2 *}$, X.-Y. Wang ${ }^{1}$, M. Lin ${ }^{1}$, Y. Tan ${ }^{1,2}$, X.-Y. Yao ${ }^{1}$ \\ and K.-Q. $\mathrm{Xu}^{1}$ \\ ${ }^{1}$ Department of Gastroenterology, \\ Changzhou Second People's Hospital Affiliated to Nanjing Medical University, \\ Changzhou, Jianshu Province, China \\ ${ }^{2}$ Department of Gastroenterology, Ningxia People's Hospital, Yinchuan, \\ Ningxia Hui Autonomous Region Province, China \\ *These authors contributed equally to this study. \\ Corresponding author: K.-Q. Xu \\ E-mail: lnlmcn@163.com
}

Genet. Mol. Res. 13 (2): 3940-3946 (2014)

Received February 15, 2013

Accepted December 17, 2013

Published May 23, 2014

DOI http://dx.doi.org/10.4238/2014.May.23.4

\begin{abstract}
We investigated the clinical significance of RUNX3 gene expression in human pancreatic carcinoma. Five samples of pancreatic tissues and 30 samples of pancreatic cancer tissues and paracancerous tissues were collected. RUNX3 expression was detected by real-time PCR and immunohistochemistry. The relationships between clinicopathological findings and the expression of RUNX3 were analyzed. The relative quantification level of RUNX3 mRNA expression in human pancreatic carcinoma tissues and paracancerous tissues was $2.60(0.42-$ $12.82)$ and $1.02(0.19-3.58)$, respectively $(\mathrm{P}<0.05)$. The percentage of positive cells expressing RUNX3 protein in human pancreatic tissues and paracancerous tissues was $45.5 \pm 26.2$ and $6.9 \pm 6.0 \%$, respectively ( $\mathrm{P}$ $<0.01)$. The high RUNX3 group $(\mathrm{N}=9)$ with $45.5 \%$ or more of the cancer cells staining for RUNX3 and the low RUNX3 group $(\mathrm{N}=21)$ with less than $45.5 \%$ cancer cells staining for RUNX3. Low expression of RUNX3 correlated significantly with an advanced TNM stage $\left(\chi^{2}=\right.$
\end{abstract}


6.897, $\mathrm{P}=0.045)$, lymph node metastasis $\left(\chi^{2}=4.739, \mathrm{P}=0.029\right)$ and neural invasion $\left(\chi^{2}=5.44, \mathrm{P}=0.020\right)$. On the other hand, no association could be found between RUNX3 expression and clinicopathological variables including age, gender, tumor location, tumor size, tumor differentiation or the serum concentration of CEA and CA199. The expression of RUNX3 in pancreatic cancer tissues was obviously higher than that in the paracancerous tissues. Low expression of RUNX3 may have an important role in aggressiveness, lymph node metastasis and neural invasion in pancreatic cancer. In pancreatic carcinoma tissues, low expression of RUNX3 may indicate a poor prognosis.

Key words: RUNX3; Pancreatic cancer; Prognosis

\section{INTRODUCTION}

Pancreatic cancer is one of the most common gastrointestinal tumors, and it ranks in the forefront among malignant tumors in incidence and mortality, showing poor prognosis. In recent years, the incidence of pancreatic cancer in China has significantly increased and is at a level close to that in Europe and the United States. Current studies indicate that there is still a lack of effective early diagnosis for pancreatic cancer. Most patients with pancreatic cancer have metastatic disease when diagnosed, and the median survival time after diagnosis is less than six mouths. Combined application of a variety of traditional treatments, including surgery, radiotherapy and chemotherapy, has failed to significantly improve the prognosis of pancreatic cancer. Therefore, it is very necessary to further clarify the molecular mechanisms of this disease.

Human RUNT-related transcription factor 3 (RUNX3) is a member of the RUNT family. As a recently discovered tumor suppressor gene, Zaidi et al. (2002) showed that RUNX3 can function through the TGF-Smad signal pathway. Thus, RUNX3 can influence cell differentiation, cell cycle regulation, apoptosis and malignant transformation; the deletion of RUNX3 can activate the tumor gene Wnt, which can induce a gastric mucosal precancerous condition.

Chronic infection with Helicobacter pylori can inactivate the RUNX3 gene by a variety of mechanisms, and lead to gastric cancer (Ito, 2011). It has been reported that the recovery of RUNX3 expression in colon cancer patients can inhibit the growth and metastasis of colon cancer cells (Peng et al., 2008). Studies have found that RUNX3 gene is expressed only in islet cells in normal pancreatic tissue and not in pancreatic cancer cells and transferred lymphocytes, suggesting that the RUNX3 gene plays a role in the pathogenesis of pancreatic cancer (Li et al., 2004).

The purpose of this study was to investigate the mRNA and protein expression of RUNX3 in pancreatic ductal adenocarcinoma and corresponding paracancerous tissues, and to explore its role in the pathogenesis of pancreatic cancer.

\section{MATERIAL AND METHODS}

\section{Clinical data}

The data of 30 cases of pancreatic cancer patients who received surgical treatment in 
our hospital were collected from January 2006 to December 2011. All patients had not received chemotherapy or radiotherapy before surgery. The specimens collected from patients who were pathologically diagnosed with pancreatic ductal adenocarcinoma, which included 16 cases of males and 14 of females, aged 38 to 86 years old (mean age of $59 \pm 12$ ). In addition, 5 cases of normal pancreatic tissue served as controls. The specimens were kept in the laboratory of Changhai Hospital and Shanghai Second Military Medical University. (Normal pancreatic specimens were obtained from accidental death cases, after written informed consent from their families).

\section{Methods}

\section{Reagents and apparatus}

Real-time PCR Master Mix was provided by TOYOBO (Japan). The Trizol kit was provided by Invitrogen (United States). The RNA extraction and RT-PCR kits were purchased from TaKaRa (Japan), and Taq DNA polymerase was purchased from Tektronix (China). 7500 Real-Time PCR instrument was purchased from ABI (United States). The primers were synthesized by Shanghai Biological Engineering (China). Mouse anti-human/rabbit universal antibody, HRP, was purchased from Long Island (China), and rabbit anti-human RUNX3 monoclonal antibodies were purchased from Beijing Boaosen Biotechnology (China).

\section{Immunohistochemical localization of RUNX3 in pancreatic tissue}

Paraffin sections were deparaffinized to water by a routine method, and $1 \% \mathrm{H}_{2} \mathrm{O}_{2}$ was used to inhibit endogenous peroxidase. The sections were then heated for antigen retrieval and cooled to room temperature. The primary antibody was added to sections, with overnight incubation at $4^{\circ} \mathrm{C}$, and HRP secondary antibody was then added to sections.

\section{The detection of pancreatic tissue RUNX3 mRNA by real-time quantitative PCR}

RNA extraction: Total RNA of pancreatic tissue was extracted according to the instructions of the Trizol kit. Concentration and purity were determined with a UV spectrophotometer, and then reverse transcribed into cDNA; Real-time quantitative PCR: RUNX3 expression was detected using SYBR Green PCR Master Mix. The primers used were upstream 5'-ACTGTGATGGCAGGCAATGAC-3' and downstream 5'-AATGGGTTCAGTTCCGAGGTG-3'. Amplification product size: 404 bp; $\beta$-actin primer sequence: upstream 5'-CGCGAGAAGATGACCCAGAT-3', downstream: 5'-GCACTGTGTTGGCGTACAGG-3'; amplification product size: $550 \mathrm{bp} ;{ }^{\Delta \Lambda} \mathrm{Ct}$ of RUNX3 mRNA was determined for five cases of normal pancreatic tissue as control. ${ }^{\Delta \Lambda} \mathrm{Ct}$ of RUNX3 gene expression was determined for 30 cases of pancreatic cancer tissue and paracancerous tissue and compared with control. The relative quantification (RQ) values appeared to have skewed distribution, requiring evaluation by the Mann-Whitney U test.

\section{Statistical analysis}

The SPSS13.0 software was used to evaluate the data. All data were expressed as 
means $\pm \mathrm{SE}$ or median (a quarter of a multiple of spacing). For measurement data, comparisons between groups were made by a $t$-test or Mann-Whitney $\mathrm{U}$ test, while the $\chi^{2}$ test was used for counting data. $\mathrm{P}<0.05$ was considered to be statistically significant.

\section{RESULTS}

\section{Expression of RUNX3 mRNA in pancreatic tumor and the adjacent tissues}

The RQ value of RUNX3 mRNA was 2.60 (0.42-12.82) and 1.02 (0.19-3.58) in pancreatic tumor and the adjacent tissues, respectively. There was significant differential expression of RUNX3 between the tumor and paracancerous tissues $(\mathrm{P}<0.01)$.

\section{Expression of RUNX3 protein in pancreatic tumor and the adjacent tissues}

Cells expressing RUNX3 protein accounted for $45.5 \pm 26.2 \%$ in pancreatic tumor, compared to $6.9 \pm 6.0 \%$ in the adjacent tissues. The proportion of RUNX3-positive cells differed significantly between the tumor and paracancerous tissues $(\mathrm{P}<0.01)$. According to the method used by Rikimaru et al. (2007), 45.5\% was set as a threshold for the average positive cell rate. Nine cases showed high $(>45.5 \%)$ RUNX3 expression and 21 cases showed low $(<45.5 \%)$ RUNX3 expression (Figure 1).

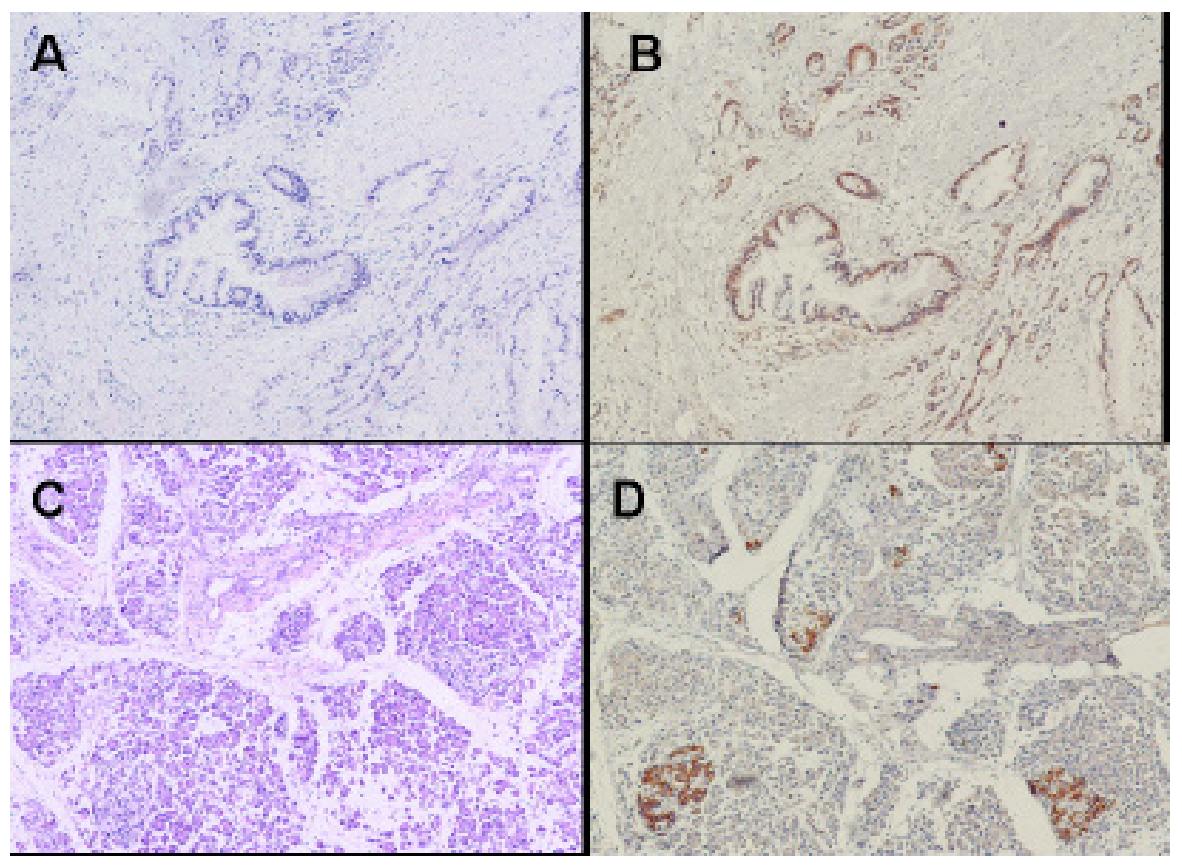

Figure 1. Expression of RUNX3 in human pancreatic cancer and paracancerous tissues. A. HE staining of pancreatic cancer. B. Expression of RUNX3 in human pancreatic cancer by SP immunohistochemical method. C. HE staining of paracancerous tissue. D. Expression of RUNX3 in human paracancerous tissue by SP immunohistochemical method (100X). 
The low expression of RUNX3 in pancreatic tumor was related to TNM stage $\left(\chi^{2}=\right.$ $6.897, \mathrm{P}=0.045)$, lymph node metastasis $\left(\chi^{2}=4.739, \mathrm{P}=0.029\right)$, perineural invasion or $\operatorname{not}\left(\chi^{2}\right.$ $=5.44, \mathrm{P}=0.020)$, but not with age, gender, tumor location, tumor size, differentiation degree, or serum CEA or CA19-9 concentration (Table 1).

\begin{tabular}{|c|c|c|c|}
\hline \multirow[t]{2}{*}{ Parameters } & \multicolumn{2}{|c|}{ RUNX3 } & \multirow[t]{2}{*}{$P$ value } \\
\hline & Low expression $(\mathrm{N}=21)$ & High expression $(\mathrm{N}=9)$ & \\
\hline Gender & & & 0.919 \\
\hline Male & 12 & 6 & \\
\hline Female & 9 & 3 & \\
\hline Age (years old) & $59.47 \pm 11.81$ & $59.18 \pm 13.11$ & 0.938 \\
\hline Tumor location & & & 0.835 \\
\hline Pancreatic head & 16 & 6 & \\
\hline Body and tail of the pancreas & 5 & 3 & \\
\hline Tumor size $(\mathrm{cm})$ & $4.00(3.25-4.75)$ & $3.50(3.13-3.88)$ & 0.213 \\
\hline Clinical stage & & & 0.045 \\
\hline I & 2 & 4 & \\
\hline II & 4 & 2 & \\
\hline III & 15 & 3 & \\
\hline Differentiation degree & & & 0.311 \\
\hline Low malignant & 3 & 3 & \\
\hline Moderate malignant & 14 & 4 & \\
\hline High malignant & 4 & 2 & \\
\hline Lymph node metastasis & & & 0.029 \\
\hline Yes & 15 & 3 & \\
\hline No & 6 & 6 & \\
\hline Perineural invasion & & & 0.02 \\
\hline Yes & 15 & 3 & \\
\hline No & 6 & 6 & \\
\hline CEA (ng/mL) & $5.00(1.99-12.59)$ & $2.85(1.80-5.55)$ & 0.244 \\
\hline CA199 (ng/mL) & $173.20(6.11-79.85)$ & $320.30(46.01-815.55)$ & 0.725 \\
\hline
\end{tabular}

\section{DISCUSSION}

Pancreatic carcinoma is a highly malignant tumor with poor prognosis, which is related to its easy invasion and metastasis. However, the pathogenesis of pancreatic carcinoma has not been fully elucidated. Tumor suppressor genes have been reported to negatively regulate carcinogenesis, development, growth, proliferation, and differentiation of pancreatic cancer cells. Tp53 and Smad/DPC4 can potentially repress tumor growth, and their functional inactivation could lead to malignant transformation and tumorigenesis (Rusiecki et al., 2011; Otto et al., 2012). RUNX3 is a recently discovered tumor suppressor gene. The human RUNX3 gene was discovered in 1994, located on lp36.1, and its length is $\sim 67 \mathrm{~kb}$ containing two promoters ( $\mathrm{P} 1$ and $\mathrm{P} 2$ ) and six exons. The RUNX3 transcription is mainly regulated by promoter $\mathrm{P} 2$ which is located before the second exon and is surrounded by a long $\mathrm{CpG}$ island. The highly conserved $\mathrm{P} 2$ promoter region is GC-rich. The protein product of RUNX3 is a heterodimer of alpha and beta subunits containing 415 amino acid residues. The $\alpha$ subunit contains a conserved RD (runt domain) composed of 128 amino acids. RD is located in the amino-terminal end of RUNX3, which contains an S-shaped immunoglobulin fold, and mediates the binding of RUNX to target DNA motif PyGPyGGT, as well as protein interactions. Furthermore, RUNX3 could directly combine with core binding factor- $\beta$ (CBF- $\beta$ ) and 
mediate the interaction between RUNX and CBF- $\beta$. The $\beta$ subunit can enhance the binding of RD to target DNA. The binding of target DNA not only can prevent the ubiquitination of RD but also maintain the stability of RUNX proteins and form a heterodimer with it, thereby increasing the affinity between RUNX proteins and DNA rather than directly making contact with DNA (Tokumaru et al., 2003).

TGF- $\beta$ is an effective inhibitor for the growth of many cells. The ligands, receptors and intracellular signal transduction molecule Smad proteins in the TGF-beta signal transduction pathway form a pathway to inhibit tumor signaling. Any abnormality in one component in the pathway can cause signal disturbance, and impact the cell biological effects of TGF- $\beta$, especially in the growth inhibitory effect for epithelial cell, which promotes the occurrence and development of tumors. Smad proteins play a very important role in this process (Ito et al., 2003). In the TGF- $\beta$ signaling transduction pathway, activated Smad complexes can specifically target sites in the nucleus from the cytoplasm and activate the targeted genes with the guidance of the RUNX3 protein, and thus regulate cell differentiation cycle, apoptosis and malignant transformation (Zaidi et al., 2002).

The activity of RUNX is controlled by various signaling pathways. Phosphorylation, acetylation, and other modifications are very important for the post-translational modification of RUNX3, which affects its stability and activity and plays an important role in the regulation of promoter methylation in RUNX3 gene, DNA repair, and genetic stability. Aberrant promoter methylation will hinder the interaction between RUNX3 transcription factor and DNA, inhibit transcription, induce gene expression related to cell proliferation and abnormal differentiation, disrupt the regulation of normal differentiation, and finally lead to the tumor development (Kato et al., 2003). The present study showed that RUNX3 expression is related to the development of a variety of malignancies, such as stomach cancer, colon cancer, ovarian cancer and liver cancer (Park et al., 2005; Chen et al., 2011; Lee et al., 2011; Sugai et al., 2011). The mechanism may be primarily through its promoter methylation or gene deletions which lead to the inactivation of theRUNX3 gene. Abnormal TGF- $\beta$ signaling pathway will cause tumor occurrence by influencing cell differentiation, cell cycle control, apoptosis and malignant transformation (Miyazono et al., 2004).

The results of our study showed that the mRNA and protein expression of RUNX3 in pancreatic carcinoma was increased compared with paracancerous tissues. There was a significant difference $(\mathrm{P}<0.05)$ between these groups, suggesting that RUNX3 has a relationship with pancreatic cancer occurrence and development. Making $45.5 \%$ of average positive cell rate the cutoff, further study showed that the low expression of RUNX3 in pancreatic cancer was related to TNM stage, lymph node metastasis and neural invasion but not to gender, tumor location, size, differentiation, or serum CEA and CA199 concentrations, which suggested that RUNX3 expression level is well connected to the characteristics and prognosis of pancreatic cancer.

In conclusion, RUNX3 expression in pancreatic cancer tissues was significantly increased compared with that in the adjacent normal tissues. The low expression of RUNX3 in pancreatic cancer tissues plays an important role in pancreatic cancer invasion, lymph node metastasis and neural infiltration. RUNX3 gene expression in pancreatic cancer reveals the degree of malignant pancreatic cancer related to invasion and metastasis. Therefore, detection of RUNX3 expression may contribute to the diagnosis and prognosis of pancreatic cancer. 


\section{REFERENCES}

Chen Y, Wei X, Guo C, Jin H, et al. (2011). Runx3 suppresses gastric cancer metastasis through inactivation of MMP9 by upregulation of TIMP-1. Int. J. Cancer 129: 1586-1598.

Ito K (2011). RUNX3 in oncogenic and anti-oncogenic signaling in gastrointestinal cancers. J. Cell Biochem. 112: 12431249.

Ito T, Shimada Y, Hashimoto Y, Kaganoi J, et al. (2003). Involvement of TSLC1 in progression of esophageal squamous cell carcinoma. Cancer Res. 63: 6320-6326.

Kato N, Tamura G, Fukase M, Shibuya H, et al. (2003). Hypermethylation of the RUNX3 gene promoter in testicular yolk sac tumor of infants. Am. J. Pathol. 163: 387-391.

Lee CW, Chuang LS, Kimura S, Lai SK, et al. (2011). RUNX3 functions as an oncogene in ovarian cancer. Gynecol. Oncol. 122: 410-417.

Li J, Kleeff J, Guweidhi A, Esposito I, et al. (2004). RUNX3 expression in primary and metastatic pancreatic cancer. $J$. Clin. Pathol. 57: 294-299.

Miyazono K, Maeda S and Imamura T (2004). Coordinate regulation of cell growth and differentiation by TGF-beta superfamily and Runx proteins. Oncogene 23: 4232-4237.

Otto N, Schulz P, Scholz A, Hauff P, et al. (2012). The proline TP53 variant stimulates likely lymphangiogenesis in an orthotopic mouse model of pancreatic cancer. Br. J. Cancer 106: 348-357.

Park WS, Cho YG, Kim CJ, Song JH, et al. (2005). Hypermethylation of the RUNX3 gene in hepatocellular carcinoma. Exp. Mol. Med. 37: 276-281.

Peng Z, Tang H, Wang X, Zhou C, et al. (2008). Inhibition of the growth and metastasis of human colon cancer by restoration of RUNX3 expression in cancer cells. Int. J. Oncol. 33: 979-984.

Rikimaru T, Taketomi A, Yamashita Y, Shirabe K, et al. (2007). Clinical significance of histone deacetylase 1 expression in patients with hepatocellular carcinoma. Oncology 72: 69-74.

Rusiecki JA, Al-Nabhani M, Tarantini L, Chen L, et al. (2011). Global DNA methylation and tumor suppressor gene promoter methylation and gastric cancer risk in an Omani Arab population. Epigenomics 3: 417-429.

Sugai M, Aoki K, Osato M, Nambu Y, et al. (2011). Runx3 is required for full activation of regulatory T cells to prevent colitis-associated tumor formation. J. Immunol. 186: 6515-6520.

Tokumaru Y, Nomoto S, Jeronimo C, Henrique R, et al. (2003). Biallelic inactivation of the RIZ1 gene in human gastric cancer. Oncogene 22: 6954-6958.

Zaidi SK, Sullivan AJ, van Wijnen AJ, Stein JL, et al. (2002). Integration of Runx and Smad regulatory signals at transcriptionally active subnuclear sites. Proc. Natl. Acad. Sci. U. S. A. 99: 8048-8053. 